\title{
Development of geometric formulation of elasticity
}

DOI:

10.1007/978-3-319-91989-8_58

\section{Document Version}

Accepted author manuscript

Link to publication record in Manchester Research Explorer

\section{Citation for published version (APA):}

Kosmas, O., \& Jivkov, A. (2018). Development of geometric formulation of elasticity. In Proceedings of the 1st International Conference on Theoretical, Applied, Experimental Mechanics (pp. 262-267)

https://doi.org/10.1007/978-3-319-91989-8_58

\section{Published in:}

Proceedings of the 1st International Conference on Theoretical, Applied, Experimental Mechanics

\section{Citing this paper}

Please note that where the full-text provided on Manchester Research Explorer is the Author Accepted Manuscript or Proof version this may differ from the final Published version. If citing, it is advised that you check and use the publisher's definitive version.

\section{General rights}

Copyright and moral rights for the publications made accessible in the Research Explorer are retained by the authors and/or other copyright owners and it is a condition of accessing publications that users recognise and abide by the legal requirements associated with these rights.

\section{Takedown policy}

If you believe that this document breaches copyright please refer to the University of Manchester's Takedown Procedures [http://man.ac.uk/04Y6Bo] or contact uml.scholarlycommunications@manchester.ac.uk providing relevant details, so we can investigate your claim.

\section{OPEN ACCESS}




\title{
Development of geometric formulation of elasticity
}

\author{
Odysseas Kosmas and Andrey Jivkov \\ School of Mechanical, Aerospace and Civil Engineering \\ University of Manchester, Manchester UK \\ odysseas.kosmas@manchester.ac.uk, andrey.jivkov@manchester.ac.uk
}

\begin{abstract}
Recently, new techniques have been presented that discretize continuous elasticity variables as cochains over a primal mesh, representing the solid, and an appropriately defined dual one. Discrete strain and stress can be then thought of as a vector-valued 1-form (or vectorvalued 1-cochain on the primal mesh) and covector-valued 2-form (or vector-valued 2-cochain on the dual mesh) respectively. The governing equations can be formulated by requiring energy balance and invariance under time-dependent rigid translations and rotations of the ambient space. To obtain those, we project the discrete stress into normal and tangential components and formulate the boundary value problem with a system of two matrix equations. This allow for treating both classical and coupled-stress (micro-polar) elasticity. The link between discrete strains and stresses is provided by a material discrete Hodge star operator, which we define to include geometric and physical factors, such as lengths, areas, and moduli of elasticity and rigidity. The performance of the proposed formulation is demonstrated by a simple example.
\end{abstract}

Keywords: discrete exterior calculus, geometric mechanics, mixed method, primal and dual mesh, discrete Hodge star

\section{Introduction}

Focusing on problems of elasticity, for the cases when the ambient space is Euclidean, different methods have been presented in order to derive the (discrete) linear systems of equations, that can be then solved by existing numerical methods. In order to study those discretization methods for partial differential equations (PDEs) the use of exterior calculus must be invoked. With that, one can define vector calculus to higher dimensions and to smooth manifolds [1]. But in order for someone to define the discretized quantities needed, Discrete Exterior Calculus (DEC) must be introduced. This way numerical methods for solving PDEs on simplicial complexes (triangle, tetrahedral or higher dimensional simplicial meshes) can be obtained $[8,5]$. Using DEC for discretization of differential operators has been formulated and then employed by an increasing number of authors in recent years to create mimetic operators [4], develop multigrid solvers $[3]$ and for the solution of various mechanics and physics problems $[9,6,10]$. 
In this work, we follow the techniques that have been presented lately, which discretize continuous elasticity variables as cochains over a primal mesh that represents the solid $[8,5]$ for the cases where the primal and dual meshes are orthonormal. Therefore we also define discrete strain and stress as a vector-valued 1 -form and covector-valued 2-form respectively. However, we split (project) the discrete stress into two components, a normal and a tangential one. Doing so, the governing equations, which can be established by requiring energy balance invariance under time-dependent rigid translations and rotations of the ambient space, refer directly to these components. As a result, the equations have now a more general applicability than the one in [10] which can be considered as a constrained system, since to that the authors force the discrete stress to be normal to each face fo the dual cell. Specifically, the equations can be used to represent both classical and coupled-stress elasticity. In the following, we first discuss the case of orthonormal primal and dual mesh, where the discrete Hodge star operator is a geometric diagonal matrix. This is then complemented by material parameters to capture the elastic behaviour of solids.

\section{Basics of DEC with reference to elasticity}

In linearized elasticity the basic unknown is the displacement field, which is a vector field on the reference configuration of the elastic body, Fig. (1). For the general case, in order to fully represent a topological space we consider that it can be a decomposition of simple pieces i.e. complexes $K$, which, in $\mathbb{R}^{3}$ involve lengths, areas and volumes. To define calculus on those complexes, a sign notation must be introduced. That can be though of as a reference orientation of any simplex $\sigma^{k}$, see for example [8]. The resulting oriented simplicial complex $\sigma^{k}$ forms then a manifold, which, for the present, we define as a completely wellcentered tetrahedral mesh, i.e. one in which the circumcenters are contained within the corresponding simplices $[8,5,10]$.

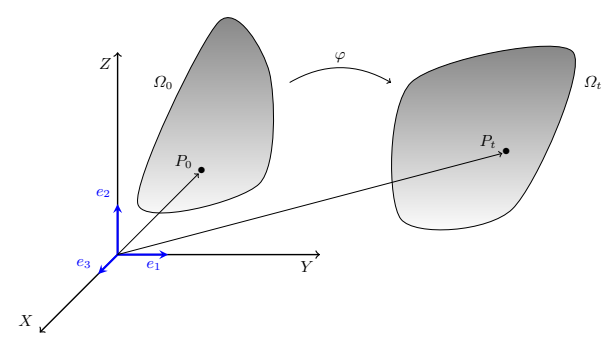

Fig. 1: Initial and deformed configuration of a continuum.

Furthermore, to use those simplicial complexes as data structures, we need to define simplicial maps that approximate continuous maps, see [8]. To that end, we also use the $k$-chains and $k$-cochains on $K$, namely we define a function $c$ with 
$c\left(-\sigma^{k}\right)=-c\left(\sigma^{k}\right)$ as a $p$-chain of $K$ for the above oriented $k$-simplices, while we will use $C_{k}(K)$ for the space of $p$-chains. From the other hand, homomorphisms of $C_{k}(K)$ to $\mathbb{R}$ are called $p$-cochains of $K$, denoted $C^{k}(K ; \mathbb{R})$ or $\operatorname{Hom}\left(C_{k}(K), \mathbb{R}\right)$. The dual $p$-cochains are denoted $C^{k}(\star K ; \mathbb{R})$, where $\star K$ is the the dual complex of $K$. The discretization map from space of smooth $p$-forms to $p$-cochains is called the de Rham map [5]

$$
R: C^{k}(K) \rightarrow C^{k}(K ; \mathbb{R}) .
$$

That can be similarly defined for the dual one using $\star K$ to the later expression.

For elasticity problems, in order to discretize the operators needed, we first define the discrete exterior derivative $d^{k}$. That can be using the coboundary operator, which as a metric independent operator for $K$, defines $d^{k}$ as $[5,7]$

$$
d^{k}: C^{k}(K) \rightarrow C^{k+1}(K) .
$$

The above operator acts on differential $k$-forms, while the sequence

$$
0 \leftarrow C^{n}(K) \stackrel{d^{n-1}}{\longleftarrow} \cdots \stackrel{d^{k}}{\longleftarrow} C^{k}(K) \stackrel{d^{k-1}}{\longleftarrow} \cdots \stackrel{d^{0}}{\longleftarrow} C^{0}(K) \leftarrow 0
$$

is called the cochain complex induced by $d^{k}$. Similarly, one can define discrete exterior derivative over a dual mesh or dual discrete exterior derivate

$$
d^{k}: C^{k}(\star K) \rightarrow C^{k+1}(\star K) .
$$

Discrete operators can be determined by the incidence structure of the given simplicial mesh. The grad, curl and divergence can be defined using incidence matrices $M_{i}^{j}$ which encode the connectivity information of a simplicial complex. We also use $\sigma^{0}(t)$ to describe a zero cell (at any time $t$ ) and its position vector in the Euclidean ambient space and $\sigma^{j}(t)$ for $j \in \mathbb{Z}$ to describe the $j$-th cell. Using those we can define the incidence matrices $[7]$

$$
M_{i}^{j}=\left\{\begin{array}{cl}
1 & \text { if } \sigma^{i} \text { is coherent with the induced orientation of } \sigma^{j} \\
-1 & \text { if } \sigma^{i} \text { is not coherent with the induced orientation of } \sigma^{j} \\
0 & \text { if } \sigma^{i} \text { is not on the boundary of } \sigma^{j}
\end{array}\right.
$$

for the cells $\sigma^{i}$ and $\sigma^{j}$ with $i, j \in \mathbb{Z}$ and $0 \leq i<j \leq 1$. Same way we define incidence matrices for the dual mesh by

$$
M_{j}^{i}=\left\{\begin{array}{cl}
1 & \text { if } \star \sigma^{i} \text { is coherent with the induced orientation of } \star \sigma^{j} \\
-1 & \text { if } \star \sigma^{i} \text { is not coherent with the induced orientation of } \star \sigma^{j} \\
0 & \text { if } \star \sigma^{i} \text { is not on the boundary of } \star \sigma^{j}
\end{array}\right.
$$

For the cases when the primal and the dual mesh are orthonormal it can be shown that $M_{i}^{j}=\left(M_{i}^{j}\right)^{T}[7]$.

Given the definition of an inner product and corresponding metric tensor, the discrete Hodge star can be also defined as a pure diagonal matrix with components

$$
H_{i j}^{k}=\frac{\left|\star \sigma_{i}^{k}\right|}{\left|\sigma_{i}^{k}\right|} \delta_{i j} .
$$


This operator relates $p$-cochains on the primal complex with $(n-p)$-cochains on the dual complex scaled by the inverse metric tensor, for further details see $[8$, $5,10]$ and references therein.

\section{Geometric formulation of elasticity}

Assuming that a discretized continuum is modelled by the primal $k$-simplex embedded in an oriented Euclidean ambient space as described above. We further identify a zero cell $\sigma^{0}(t)$ (at any time $t$ ) with its position vector in the Euclidean ambient space. The time dependent simplicial mapping $\phi_{t}: k \rightarrow \phi_{t}(k)$ can be regarded as the discrete deformation map $\sigma_{i}^{0}(t)=\phi_{t}\left(\sigma_{i}^{0}\right), \forall \sigma_{i} \in k^{(0)}$.

Here we define the discrete traction as a 2-cochain on the dual mesh. More precisely we define it as a covector at the barycentre $B\left(\star \sigma^{1}\right)$ of $\star \sigma^{1}$, see Figure 2. Given an orientation to primal cells for our oriented complex, discrete stress $\mathbb{t}$ associates a 2-cochain to each oriented $\star \sigma^{1}$ (where $\star \sigma^{1}$ we consider the boundary of $\star \sigma^{0}$ that for the case of tetrahedral meshes consisting of triangles, Figure 2). For the shared surface $\star \sigma^{1}$ between two neighbour $\star \sigma^{0}$ traction is considered to change sign between those dual cells, $[9,5,10]$
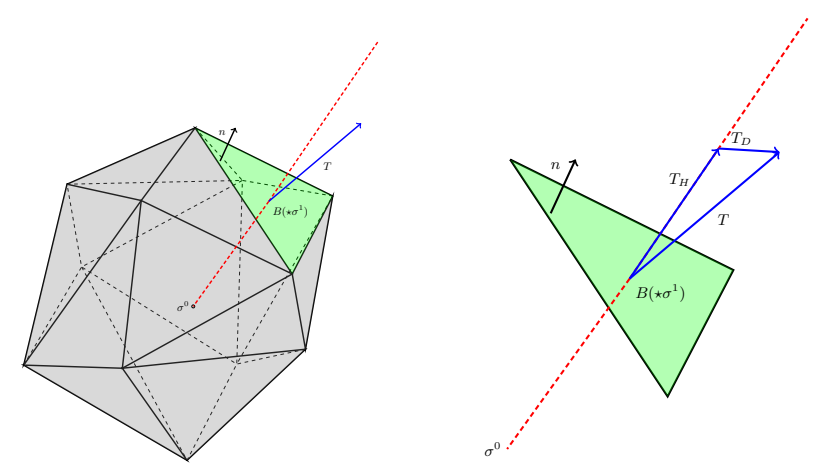

Fig. 2: The dual cell $\star \sigma^{0}$ around primal point $\sigma^{0}$ and the traction at the barycentre of $\star \sigma^{1}$ (left) and the decomposition of the traction vector $\mathbf{T}$ to a normal and a tangential one on $\star \sigma^{1}(t)$ (right).

Doing so, all balance laws can be obtained by postulating balance of energy and its invariance under rigid translations and rotations of the ambient space [5]. In order to derive those equations for the dual cell we decompose the traction vector on two components $\mathbf{T}_{H}$ at the direction of the line connecting $\star \sigma^{0}$ with $B\left(\star \sigma^{1}\right)$ and $\mathbf{T}_{D}$ that follows from the equation

$$
\mathbf{T}=\mathbf{T}_{H}+\mathbf{T}_{D}
$$

see Figure 2. 
If we use the incidence matrices described in (5) and (6), the balance of linear and angular momentum in $\mathbb{R}^{3}$ can be written in matrix form

$$
\left[\begin{array}{cc}
R M_{1}^{0} & I \\
R^{\perp} M_{1}^{0} & 0
\end{array}\right]\left[\begin{array}{l}
T \\
B
\end{array}\right]=\left[\begin{array}{l}
0 \\
0
\end{array}\right]
$$

where the matrix $T$ is the discrete stress, $B$ is the discrete body force and $I$ the identity matrix. Furthermore matrices $R$ and $R^{\perp}$ are the projection matrices of $\mathbf{T}$ to normal and tangential direction of $\star \sigma^{1}$. If we further denote $U$ the matrix of displacements the discrete stress can be written as

$$
T=H^{1} M_{1}^{0} U .
$$

The system of equations (9) can be solved in order to obtain the unknown displacements and stresses (or unknown body forces when a boundary point is fixed). Finally, in order to capture the elastic behavior of solids, we define the material discrete Hodge star $H^{k}$ using the geometric part of (10) and a tensorial term that contains the modulus of elasticity $E_{a}$ for all axial components and the modulus of rigidity $G_{b}$ for the normal ones. So we have

$$
H^{k}=\left[\begin{array}{ccc}
\frac{\left|\star \sigma_{i}^{k}\right|}{\left|\sigma_{i}^{k}\right|} & 0 & 0 \\
0 & \frac{\left|\star \sigma_{i}^{k}\right|}{\left|\sigma_{i}^{k}\right|} & 0 \\
0 & 0 & \frac{\left|\star \sigma_{i}^{k}\right|}{\left|\sigma_{i}^{k}\right|}
\end{array}\right]\left[\begin{array}{ccc}
E_{a} & 0 & 0 \\
0 & G_{b} & 0 \\
0 & 0 & G_{b}
\end{array}\right] .
$$

When barycenters are used to define the dual complex a correction factor has been proposed by [2] and could be also used to the above definition.

\section{Bending of a cantilever beam}

As a test example we present a classical solid mechanics problem, the bending of a prismatic cantilever beam. To our preliminary results we considered the beam to be completely fixed in all the directions on the left end while its cross section is $1 \times 1 \mathrm{~m}$ and its length $10 \mathrm{~m}$. We have used tetrahedral mesh as the primal one while the dual has been defined using circumcenter points of each simplex.

The loading of the right side consists of a point force of $9 \times 10^{6} N$, see Figure 3a. We further assume that the solid behaves like a Hookean solid with Young's modulus $E=5 \cdot 10^{6} \mathrm{~N} / \mathrm{m}^{2}$ and Poisson's ratio $\nu=0.35$. Finally Figure $3 \mathrm{~b}$ presents the deflection of the beam in the $z$ direction.

\section{Conclusions and perspectives}

The discrete formulation of elasticity proposed here gives the benefit of clear differentiation between the topological, geometric and physical contributions to the deformation behaviour of solids. This is the first step towards discrete modelling of more complex behaviour for engineering applications, including irreversible deformations and fracture, which is subject of ongoing work. 


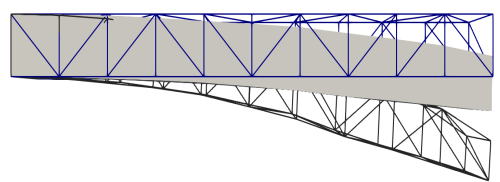

(a) Snapshots of the motion of $3 d$ cantilever beam.

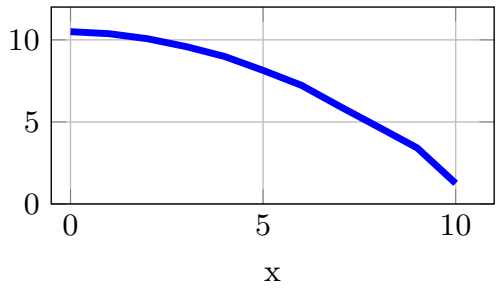

(b) Deflection in the $z$ direction.

\section{Acknowledgments}

The authors appreciate highly the support of EPSRC via grant EP/N026136/1 "Geometric Mechanics of Solids".

\section{References}

1. R. Abraham, J.E. Marsden, and R. Ratiu. Manifolds, Tensor Analysis, and Applications: 2Nd Edition. Springer-Verlag New York, Inc., New York, NY, USA, 1988.

2. B. Auchmann and S. Kurz. A geometrically defined discrete hodge operator on simplicial cells. IEEE Trans. Magn., 42:643-646, 2006.

3. N. Bell and A.N. Hirani. Pydec: Software and algorithms for discretization of exterior calculus. ACM Trans. Math. Softw., 39(1):3:1-3:41, November 2012.

4. P.B. Bochev and J.M. Hyman. Principles of Mimetic Discretizations of Differential Operators, pages 89-119. Springer New York, New York, NY, 2006.

5. M. Desbrun, A.N. Hirani, M. Leok, and J.E. Marsden. Discrete exterior calculus. ArXiv Mathematics e-prints, August 2005.

6. J. Frauendiener. Discrete differential forms in general relativity. Classical and Quantum Gravity, 23(16):S369, 2006.

7. L. Grady and J.R. Polimeni. Discrete calculus - applied analysis on graphs for computational science. 2010.

8. A.N. Hirani. Discrete Exterior Calculus. PhD thesis, California Institute of Technology, Pasadena, CA, USA, 2003. AAI3086864.

9. A.N. Hirani, K.B. Nakshatrala, and J.H. Chaudhry. Numerical method for darcy flow derived using discrete exterior calculus. International Journal of Computational Methods in Engineering Science and Mechanics, 16(3):151-169, 52015.

10. A. Yavari. On geometric discretization of elasticity. Journal of Mathematical Physics, 49(2):022901, 2008. 